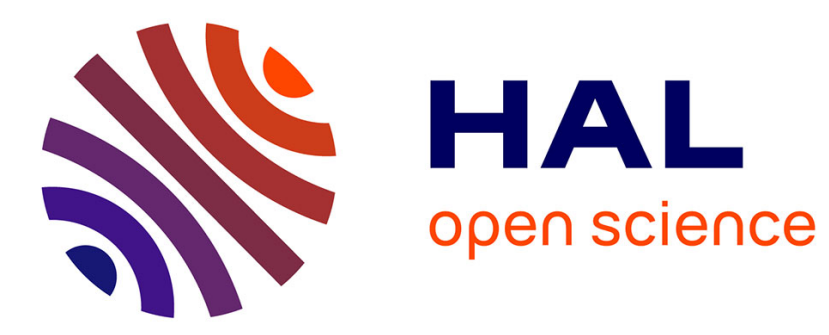

\title{
Elicitation of a Utility from Uncertainty Equivalent Without Standard Gambles
}

\author{
Christophe Labreuche, Sébastien Destercke, Brice Mayag
}

\section{To cite this version:}

Christophe Labreuche, Sébastien Destercke, Brice Mayag. Elicitation of a Utility from Uncertainty Equivalent Without Standard Gambles. European Conference on Symbolic and Quantitative Approaches to Reasoning with Uncertainty 2015, Jul 2015, Compiègne, France. pp.25-35, 10.1007/9783-319-20807-7_3. hal-01200696

\section{HAL Id: hal-01200696 \\ https://hal.science/hal-01200696}

Submitted on 17 Sep 2015

HAL is a multi-disciplinary open access archive for the deposit and dissemination of scientific research documents, whether they are published or not. The documents may come from teaching and research institutions in France or abroad, or from public or private research centers.
L'archive ouverte pluridisciplinaire HAL, est destinée au dépôt et à la diffusion de documents scientifiques de niveau recherche, publiés ou non, émanant des établissements d'enseignement et de recherche français ou étrangers, des laboratoires publics ou privés. 


\title{
Elicitation of a utility from uncertainty equivalent without standard gambles
}

\author{
Christophe Labreuche ${ }^{1}$, Sébastien Destercke ${ }^{2}$, Brice Mayag ${ }^{3}$ \\ ${ }^{1}$ Thales Research \& Technology, Palaiseau, France \\ christophe. labreuchedthalesgroup.com \\ ${ }^{2}$ Heudiasyc, Université de Technologie de Compiègne, Compiègne, France \\ sebastien. destercke@hds.utc.fr \\ ${ }^{3}$ LAMSADE, University of Paris Dauphine, Paris, France \\ brice.mayag@dauphine.fr
}

\begin{abstract}
In the context of decision under uncertainty, standard gambles are classically used to elicit a utility function on a set $X$ of consequences. The utility of an element $x$ in $X$ is derived from the probability $p$ for which a gamble giving the best outcome in $X$ with probability $p$ and the worst outcome in $X$ otherwise, is indifferent to getting $x$ for sure. In many situations, uncertainty that can be observed on the true value of $X$ concerns only neighbour values. Uncertainty is then represented by a probability distribution whose support is an interval. In this case, standard gambles are unrealistic for the decision maker. We consider uncertainty represented by an equi-probability over an interval of $X$. This paper addresses the elicitation of a utility function on $X$ by obtaining the certainty equivalent of an equi-probability over an interval of $X$. We show that not all utility models are suitable to accomplish this task.
\end{abstract}

\section{Introduction}

The elicitation of a utility function $u$ over a set $X$ is an important aspect of decision theory. It can be performed in decision under uncertainty by observing the attitude of the decision maker towards risk over gambles defined on $X$ [18]. The most classical way to elicit $u$ is based on standard gambles. A standard gamble (or standard lottery) denotes a vector $\left\langle p, x^{\top} ; 1-p, x^{\perp}\right\rangle$ where the best outcome $x^{\top}$ (resp. the worst outcome $x^{\perp}$ ) in $X$ is realized with probability $p$ (resp. $1-p$ ). For some $x \in X$, one gets from the decision maker the probability $p$ for which the standard gamble $\left\langle p, x^{\top} ; 1-p, x^{\perp}\right\rangle$ is indifferent to the sure outcome $x$. Under the expected utility (EU) model, one obtains $u(x)=p$, after fixing $u\left(x^{\top}\right)=1$ and $u\left(x^{\perp}\right)=0[18,19]$. This elicitation approach has been used for instance to construct the utility of the remaining years to live, for medical decisions. Such a gamble can be a $50-50$ gamble resulting in either 20 years of good health or immediate death [20].

The idea of the previous approach is to elicit $u(x)$ by identifying an uncertain situation (a probability distribution over the set $X$ of consequences) that has $x$ as certainty equivalent. The uncertain situation is then a standard gamble based on the extreme consequences $x^{\top}$ and $x^{\perp}$. There are many applications where standard gambles do not make sense to the decision maker. Let us consider the following example. 
Example 1. In crisis management, if heavy rain is expected, the local authority would like to forecast the peak flood level in a city. Before the flood arises, the decision maker only has an uncertain estimate of the peak flood level $X$. The problem is then to define a utility function on this variable, to be combined with other criteria to make a decision on the evacuation of a residential area. The flood propagation models typically return an extreme value distribution.

In the previous example, it might not be easy to elicit the utility function on $X$ on the basis of the distribution on the peak flood level provided by the models as it is relatively complex. We note that this distribution has a support which is a closed interval of $X$. Hence it would not be realistic to use standard gambles, like $\langle p, 15 m ; 1-p, 0 m\rangle$, as the decision maker will not face such a situation in a real crisis management. We propose in this paper to use uniform distribution law on a close interval like $[10 \mathrm{~m}, 14 \mathrm{~m}]$. The uniform law can be seen as an approximation of the extreme value distribution, which is simple to grasp for a decision maker. We restrict ourselves to uniform probability laws over intervals of $X$, such as a uniform probability on $[10 m, 14 m]$ in Ex. 1 .

We are interested in constructing a utility function on $X$ from the certainty equivalent $\widehat{x}$ of a uniform probability law on an interval $[a, b]$ of $X$. Utility functions are parameterized for elicitation purposes. The certainty equivalent $\widehat{x}$ can potentially be any element in interval $[a, b]$. Then a family of parameterized utility functions is admissible if, for any $\widehat{x} \in[a, b]$, there exists a value of the parameters for which the expected value of the utility function over $[a, b]$ is equal to $\widehat{x}$. We show that the most commonly used models do not fulfilled this requirement. We propose some models that satisfy it.

In practice, one cannot expect to identify accurately the certainty equivalent of a probability law over $X$. Hence we do not obtain a unique utility function but rather a family of compatible utility functions, from which decisions are to be taken. We adopt a cautious approach to recommend decisions $[8,16]$.

Section 2 presents the general elicitation approach. We address in Section 3 piecewise affine utility functions, which is a commonly used representation in multi-criteria decision making. We then consider in Section 4 an analytical formula, as it is done in decision under uncertainty. Section 5 presents the related works. Finally some conclusions are drawn.

\section{General approach for the elicitation of a utility function}

Let $X$ be an interval of $\mathbb{R}$. Without loss of generality, we will consider in the whole paper only strictly increasing utility functions over $X$. In an elicitation phase, one cannot expect to uniquely identify the utility function. Hence we assume a family $U$ of compatible utility functions, where $U$ is to be determined.

\subsection{Decision model under uncertainty}

We define a gamble on $X$ as a probability density function on $X$. We wish to represent a preference relation over these gambles, given the set $U$. Here two gambles describe two different uncertainties on $X$, and we are interested in the attitude of the decision 
maker toward such uncertainty. Note that this definition of a gamble is different from that used in subjective probability $[6,22]$, where a gamble is a reward associated to each state of nature.

The basic decision rule in decision under uncertainty is based on expected utility:

$$
\mathrm{EU}_{u}(g)=\int_{X} u(x) p(x) d x
$$

where $p$ is the probability density function associated to gamble $g$. There are many different decision rules to compare gambles when the parameters of the model are imprecise: [21] for imprecise probabilities, [8] for imprecise utilities, and [16] for both imprecise probabilities and utilities. Lower and upper expectations are often used. We use a cautious way to make a decision on the gambles, facing $U$ (imprecise utilities), where the relation holds if the preference is true for all utility functions in $U$ :

$$
\begin{aligned}
& g \succsim_{U} g^{\prime}\left(\text { resp. } g \succ_{U} g^{\prime} \text { or } g \sim_{U} g^{\prime}\right) \quad \Longleftrightarrow \\
& \forall u \in U, \operatorname{EU}_{u}(g) \operatorname{EU}_{u}\left(g^{\prime}\right)\left(\text { resp. } \operatorname{EU}_{u}(g)>\operatorname{EU}_{u}\left(g^{\prime}\right) \text { or } \operatorname{EU}_{u}(g)=\operatorname{EU}_{u}\left(g^{\prime}\right)\right)
\end{aligned}
$$

Relation induced by $\succsim_{U}$ is usually incomplete.

\subsection{Elicitation process}

Once the utility is known, the decision model $\succsim_{U}$ can be applied to probability laws $p$ that are very complex (as for the flood peak level in Ex. 1). However, during the elicitation process, we restrict ourselves to uniform probability laws over intervals of $X$ in order to reduce the cognitive load. We denote by $\langle 1,[a, b]\rangle$ (with $[a, b] \subseteq X$ and $b>a$ ) the gamble described by the uniform probability density function $p$ given by $p(x)=\frac{1}{b-a}$ if $x \in[a, b]$ and $p(x)=0$ else. The sure outcome $x \in X$ is also noted $\langle 1,[x, x]\rangle$. We set $\mathcal{G}_{X}=\{\langle 1,[a, b]\rangle,[a, b] \subseteq X\}$ including both cases. We have $\mathrm{EU}_{u}(\langle 1,[a, b]\rangle)=\frac{1}{b-a} \int_{a}^{b} u(x) d x$ if $b>a$, and $\mathrm{EU}_{u}(\langle 1,[a, b]\rangle)=u(a)$ if $a=b$.

In order to ease the elicitation process, we are interested in families of parameterized utility functions. This is classically done in decision under uncertainty, with for instance family $u_{\lambda}(x)=x^{\lambda}[13,17]$. We denote by $\gamma$ the vector of parameters, by $\Gamma$ its range, and by $u_{\gamma}$ the associated utility function. Let $\mathcal{U}=\left\{u_{\gamma}, \gamma \in \Gamma\right\}$. The set of admissible utility functions corresponds to a subset $\Gamma_{A}$ of $\Gamma$, where $U=\left\{u_{\gamma}, \gamma \in \Gamma_{A}\right\}$.

Generalizing the elicitation process based on standard gambles, $\Gamma_{A}$ may be derived by asking to the decision maker the certainty equivalent $\widehat{x}$ of a gamble $\langle 1,[a, b]\rangle$, given interval $[a, b]$. The certainty equivalent of gamble $\langle 1,[a, b]\rangle$ is an element $\widehat{x} \in[a, b]$ such that $\langle 1,[a, b]\rangle$ is indifferent to $\langle 1,[\widehat{x}, \widehat{x}]\rangle$. Then $\Gamma_{A}$ is the set of values $\gamma$ satisfying relation $\frac{1}{b-a} \int_{a}^{b} u_{\gamma}(x) d x=u_{\gamma}(\widehat{x})$.

In practice, a decision maker is not expected to provide a value $\widehat{x}$ that is close to the extreme values $a$ and $b$. Hence one might often have

$$
\langle 1,[a+\varepsilon, a+\varepsilon]\rangle \prec_{U}\langle 1,[a, b]\rangle \prec_{U}\langle 1,[b-\varepsilon, b-\varepsilon]\rangle
$$

for some $\varepsilon>0$ which depends on the attitude of the decision maker. Note that $\varepsilon$ can be very small if the decision maker is extremely risk averse or risk seeking. We will show 
in Section 3.1, that a classical family of utility functions satisfies (2) with $\varepsilon=\frac{b-a}{4}$. This value is relatively large (only half of interval $[a, b]$ is reachable), and we guess that this family is not versatile enough.

As it is not easy to set some value for $\varepsilon$ and we do not want to rule out some extreme attitudes of decision makers, we would like to ideally to represent the case where the certainty equivalent of gamble $\langle 1,[a, b]\rangle$ can be any element in the open interval $(a, b)$.

Condition $\operatorname{Comp}(a, b)$ - Completeness (with $b>a$ ): For every $\bar{x} \in(a, b)$,

$$
\exists \gamma \in \Gamma \quad \frac{1}{b-a} \int_{a}^{b} u_{\gamma}(x) d x=u_{\gamma}(\bar{x}) .
$$

Conversely, from the intermediate value theorem, we know that if function $u_{\gamma}$ is continuous, then for every $\gamma \in \Gamma$, there exists a point $\bar{x} \in[a, b]$ such that (3) holds.

One can readily see that if function $u_{\gamma}$ is constant, then condition $\operatorname{Comp}(a, b)$ is trivially satisfied for every interval $[a, b]$. Hence we consider henceforth only strictly increasing utility functions.

In practice, it is realistic to ask directly to the decision to provide the value of the certainty equivalent of a gamble. The certainty equivalent $\widehat{x}$ can be approximated, by asking questions of the following form (with $\langle 1,[a, b]\rangle \in \mathcal{G}_{X}$ and $\bar{x} \in(a, b)$ )

"Is $\langle 1,[a, b]\rangle$ less preferred / preferred / indifferent / incomparable to $\langle 1,[\bar{x}, \bar{x}]\rangle$ ?'

for different values of $\bar{x}$, proceeding by dichotomy on $\bar{x}$. The so-obtained dichotomy process for approximating $\widehat{x}$ given gamble $\langle 1,[a, b]\rangle$ is called Certainty Equivalent Estimate (CEE). If the answer is "less preferred" (resp. "preferred" or "indifferent"), then for all $\gamma \in \Gamma_{A}, \mathrm{EU}_{u_{\gamma}}(\langle 1,[a, b]\rangle)-u_{\gamma}(\bar{x})<0$ (resp. $>0$ or $\left.=0\right)$. At the end, $\Gamma_{A}$ is the set of all values $\gamma$ satisfying these constraints. "Incomparability" answers are not explicitly represented as constraints.

The remaining of this paper is devoted to finding models of utility that fulfil Comp. We will see that condition Comp is not fulfilled with the most commonly used classes of utility functions. This condition will be used to select suitable families $\mathcal{U}$.

\section{Case of piecewise affine utility functions}

Piecewise affine utility functions are classically used in multi-criteria decision making [1]. The decision maker provides a finite set of elements in $X: x_{1}<x_{2}<\cdots<x_{m}$. We set

$$
u_{\gamma}\left(x_{1}\right)=0 \quad \text { and } \quad u_{\gamma}\left(x_{m}\right)=1 \text {. }
$$

The unknowns are the utility at the points $x_{2}, \ldots, x_{m-1}: \gamma=\left(u_{2}, \ldots, u_{m-1}\right)$, where $u_{k}=u_{\gamma}\left(x_{k}\right), u_{1}=0$ and $u_{m}=1$. As $u_{\gamma}$ is strictly increasing, we assume that $u_{1}<u_{2}<\cdots<u_{m-1}<u_{m}$. The utility function which interpolates between the points $\left(x_{1}, u_{1}\right), \ldots,\left(x_{m}, u_{m}\right)$, is denoted $u_{\gamma}^{\mathrm{PA}}$ (where PA stands for Piecewise Affine):

$$
u_{\gamma}^{\mathrm{PA}}(x)=\left\{\begin{array}{l}
0 \quad \text { if } x \leq x_{1} \\
u_{k}+\frac{x-x_{k}}{x_{k+1}-x_{k}}\left(u_{k+1}-u_{k}\right) \quad \text { if } x \in\left[x_{k}, x_{k+1}\right] \\
1 \quad \text { if } x \geq x_{m}
\end{array}\right.
$$

We first show that form (6) does not fulfill condition Comp. Then we propose another form of piecewise affine utility function. 


\subsection{Verification of condition Comp with $\boldsymbol{u}_{\gamma}^{\mathrm{PA}}$}

As the elements $x_{1}, \ldots, x_{m}$ have a special meaning to the decision maker, we can ask questions of the form (4) with the value of $a$ and $b$ being elements in $x_{1}, \ldots, x_{m}$.

Lemma 1. Condition Comp is not fulfilled with $u_{\gamma}^{\mathrm{PA}}$. More precisely, for every $p, q \in$ $\{1, \ldots, m\}$ with $q>p$, there exists $\gamma$ such that (3) holds with $a=x_{p}$ and $b=x_{q}$ iff

$$
\bar{x} \in\left[x_{p}+\left(x_{p+1}-x_{p}\right) \frac{x_{q}-\frac{1}{2} x_{p+1}-\frac{1}{2} x_{p}}{x_{q}-x_{p}}, x_{q-1}+\left(x_{q}-x_{q-1}\right) \frac{x_{q}-x_{q-1}}{2\left(x_{q}-x_{p}\right)}\right]
$$

Proofs are omitted due to space limitation. The idea is that, in order to allow having $\bar{x}$ close to the lower bound $a=x_{p}$ (resp. upper bound $b=x_{q}$ ), the utility function should be close to the Heaviside function at $x_{p}$ (resp. $x_{q}$ ) - see function $u^{1}$ (resp. $u^{2}$ ) in the right part of Fig. 1. Lemma 1 shows that this is not the case with $u_{\gamma}^{\mathrm{PA}}$. Interval in (7) is strictly included in $\left[x_{p}, x_{q}\right]$. For instance for $X=[0,1], p=1, q=m=3$ and $x_{1}=0, x_{2}=\frac{1}{2}, x_{3}=1$, interval in $(7)$ is $\left[\frac{1}{4}, \frac{3}{4}\right]$, to be compared with interval $[0,1]$. It follows that the expected utility $\mathrm{EU}_{u_{\gamma}^{\mathrm{PA}}}\left(\left\langle 1,\left[x_{p}, x_{q}\right]\right\rangle\right)$ cannot take any value in $\left[x_{p}, x_{q}\right]$. This comes from the fact that the points $x_{1}, \ldots, x_{m}$ are fixed. Hence we need to find another representation.

\subsection{Piecewise Affine function around a diagonal}

Instead of fixing the value of $x$ and letting the associated utility be a variable, the idea is to allow both the value of $x$ and its utility to be variable (but not independently).

We start by defining a utility function, depending on only one parameter $\gamma$, in an interval $[a, b]$ and with fixed values of the utility at the boundary: $u_{\gamma}(a)=c$ and $u_{\gamma}(b)=d$. The values of $a, b \in X$ and $c, d \in \mathbb{R}$ are fixed. We consider a piecewise affine function with an intermediate point in the diagonal line between $(a, d)$ and $(b, c)$ (see the left part of Figure 1). Let $\gamma \in[0,1]$; the intermediate point has coordinates $(a+\gamma(b-a), c+(1-\gamma)(d-c))$. On the whole, $u_{\gamma}$ performs an affine interpolation between the points $(a, c),(a+\gamma(b-a), c+(1-\gamma)(d-c))$ and $(b, d)$. We denote this utility function $u_{\gamma}^{\mathrm{AD}}$ (where $\mathrm{AD}$ stands for Affine around a Diagonal).

In order to elicit $\gamma$, we use the Dichotomy method CEE based on Question (4) with interval $[a, b]$. The next result shows that it completely makes sense.

Lemma 2. Condition $\operatorname{Comp}(a, b)$ is fulfilled with $u_{\gamma}^{\mathrm{AD}}$. Moreover, if $\langle 1,[a, b]\rangle$ is less preferred (resp. preferred or indifferent) to $\bar{x} \in(a, b)$, then $\gamma<\frac{\bar{x}-a}{b-a}$ (resp. $\gamma>\frac{\bar{x}-a}{b-a}$ or $\left.\gamma=\frac{\bar{x}-a}{b-a}\right)$.

The main advantage of this approach is that whatever the answer $\bar{x}$ of the decision maker in the interval $[a, b]$, one can find the value of parameter $\gamma \in[0,1]$. Moreover the correspondence between $\bar{x}$ and $\gamma$ is very simple, as the mean value of $u_{\gamma}^{\mathrm{AD}}$ is attained precisely at the breaking point $(a+\gamma(b-a), c+(1-\gamma)(d-c))$ on the diagonal. In particular, the value of $\gamma$ is independent of the values of $c$ and $d$. We will use this property in the next section, where $c$ and $d$ may be unknown.

The previous pattern can be applied only once to $a=x_{1}, b=x_{2}, c=0$ and $d=1$. 

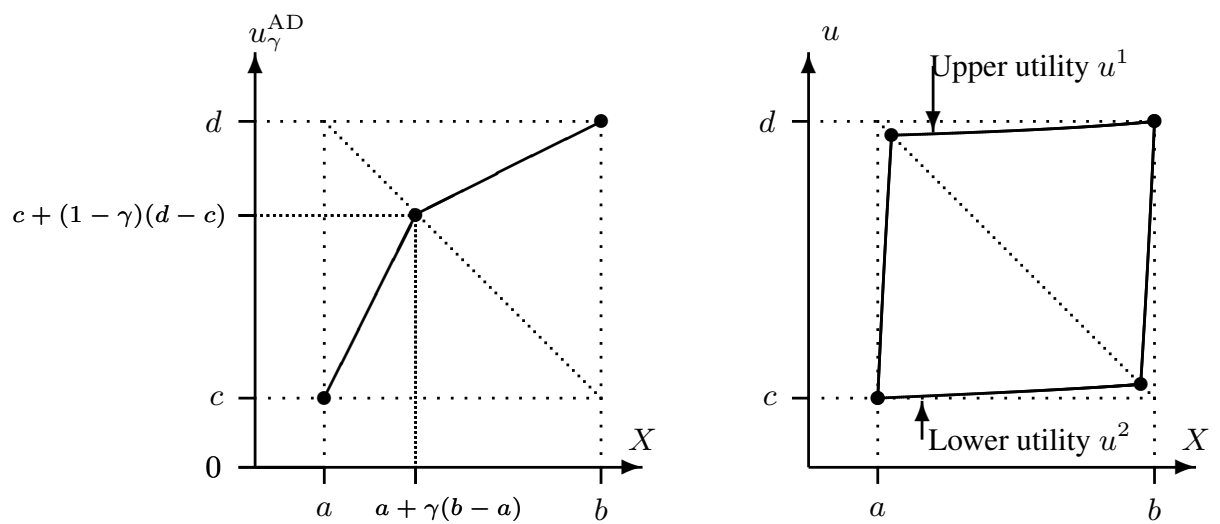

Fig. 1. Piecewise affine utility function around the diagonal $(a, d)-(b, c)$.

\subsection{Proposal with more parameters}

If we want more intermediate points, we can apply the previous patterns several times. In figure 2, we apply the pattern two times, where the three values $x_{1}, x_{2}, x_{3}$ are fixed. More precisely, we use the pattern a first time on the input interval $\left[x_{1}, x_{2}\right]$ and output interval $\left[0, u_{2}\right]$, and a second time on the input interval $\left[x_{2}, x_{3}\right]$ and output interval $\left[u_{2}, 1\right]$.

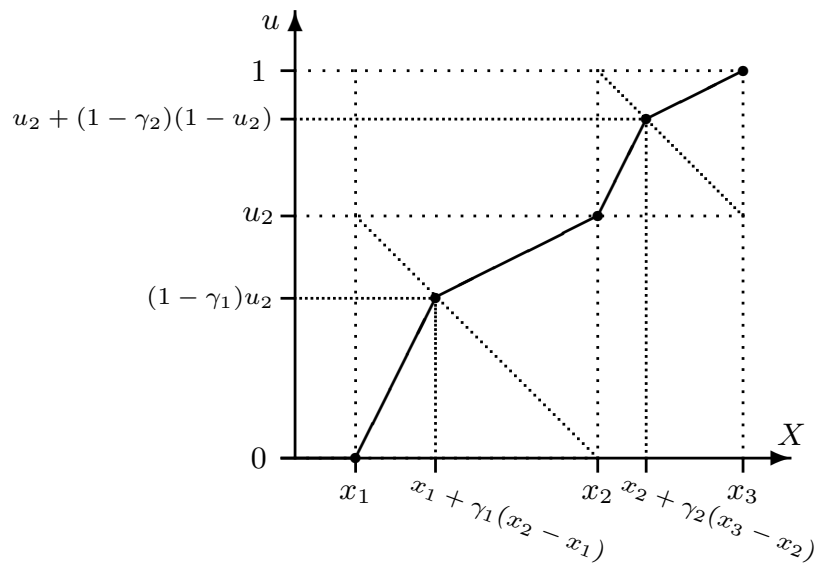

Fig. 2. Parametric piecewise affine utility function using two patterns.

We have three unknowns: $\gamma=\left(\gamma_{1}, \gamma_{2}, u_{2}\right)$. We use three times the process Dichotomy method CEE.

The first use of Dichotomy method CEE is on interval $\left[x_{1}, x_{2}\right]$. If $\left\langle 1,\left[x_{1}, x_{2}\right]\right\rangle$ is less preferred (resp. preferred or indifferent) to $\bar{x}_{1,2} \in\left[x_{1}, x_{2}\right]$, then, by Lemma 2

$$
\gamma_{1}<\frac{\bar{x}_{1,2}-x_{1}}{x_{2}-x_{1}}\left(\text { resp. } \gamma_{1}>\frac{\bar{x}_{1,2}-x_{1}}{x_{2}-x_{1}} \text { or } \gamma_{1}=\frac{\bar{x}_{1,2}-x_{1}}{x_{2}-x_{1}}\right) .
$$

The identification of $\gamma_{1}$ is independent of unknown $u_{2}$. 
The second use of Dichotomy method CEE is on interval $\left[x_{2}, x_{3}\right]$. If $\left\langle 1,\left[x_{2}, x_{3}\right]\right\rangle$ is less preferred (resp. preferred or indifferent) to $\bar{x}_{2,3} \in\left[x_{2}, x_{3}\right]$, then

$$
\gamma_{2}<\frac{\bar{x}_{2,3}-x_{2}}{x_{3}-x_{2}}\left(\text { resp. } \gamma_{2}>\frac{\bar{x}_{2,3}-x_{2}}{x_{3}-x_{2}} \text { or } \gamma_{2}=\frac{\bar{x}_{2,3}-x_{2}}{x_{3}-x_{2}}\right) .
$$

The identification of $\gamma_{2}$ is independent of unknown $u_{2}$.

Finally, the last use of Dichotomy method CEE is on the interval $\left[x_{1}, x_{3}\right]$. The decision maker is asked to compare $\left\langle 1,\left[x_{1}, x_{3}\right]\right\rangle$ with the sure outcome $\bar{x}_{1,3} \in\left[x_{1}, x_{3}\right]$. As

$$
\mathrm{EU}_{u_{\gamma}}\left(\left\langle 1,\left[x_{1}, x_{3}\right]\right\rangle\right)=\frac{x_{2}-x_{1}}{x_{3}-x_{1}}\left(1-\gamma_{1}\right) u_{2}+\frac{x_{3}-x_{2}}{x_{3}-x_{1}}\left(u_{2}+\left(1-\gamma_{2}\right)\left(1-u_{2}\right)\right),
$$

One can derive from (10) constraints on $u_{2}$, given the answer of the comparison of $\left\langle 1,\left[x_{1}, x_{3}\right]\right\rangle$ with the sure outcome $\bar{x}_{1,3}$, and upper and lower bounds on $\gamma_{1}$ and $\gamma_{2}$.

\section{Parametric utility functions}

We consider in this section parametric utility functions. We restrict ourselves to $X=$ $[0,1]$. We start with family $u_{\gamma}^{\text {pow }}(x)=x^{\gamma}$, with $\gamma>0$, already mentioned previously $[13,17]$ (see also [7] for a quadratic model). Another family will then be considered.

\subsection{Power function}

Lemma 3. Condition $\operatorname{Comp}(0,1)$ is not fulfilled with $u_{\gamma}^{\text {pow }}$. More precisely, there exists $\gamma$ such that (3) holds with $a=0$ and $b=1$ iff $\bar{x} \in\left(\frac{1}{e}, 1\right)$.

With model $u_{\gamma}^{\text {pow }}$, the decision maker is not allowed to provide a value of $\bar{x}$ outside interval $\left(\frac{1}{e}, 1\right)$. Utility function $u_{\gamma}^{\text {pow }}$ tends to the Heaviside function at 0 when $\gamma$ tends to 0 . However it does not imply that condition $\operatorname{Comp}(0,1)$ is not necessarily satisfied with $\bar{x}$ arbitrarily close to 0 . The shape of $u_{\gamma}^{\text {pow }}$ is such that its mean value $v_{\gamma}$ tends to 1 when $\gamma \rightarrow 1$, but $\left(u_{\gamma}^{\text {pow }}\right)^{-1}\left(v_{\gamma}\right)$ does not tend to 0 .

\subsection{MinMaxVar parametric function}

As Lemma 3 shows that the power utility function $u_{\gamma}^{\text {pow }}$ is not suitable, we consider another parametric function called MinMaxVar [5] taking the following expression:

$$
u_{\gamma}^{\mathrm{MMV}}(x)=1-\left(1-x^{\frac{1}{\gamma}}\right)^{\gamma}
$$

where $u_{\gamma}^{\mathrm{MMV}}(0)=0$ and $u_{\gamma}^{\mathrm{MMV}}(1)=1$ (see conditions (5)). Parameter $\gamma$ belongs to $\Gamma=(0, \infty)$, where function $u_{\gamma}^{\mathrm{MMV}}$ is convex for $\gamma<1$ and is concave for $\gamma>1$.

Function $u_{\gamma}^{\mathrm{MMV}}$ has an useful symmetry property. Indeed one can readily check that

$$
y=u_{\gamma}^{\mathrm{MMV}}(x) \quad \Longleftrightarrow \quad 1-x=u_{\gamma}^{\mathrm{MMV}}(1-y) .
$$


Hence points $(x, y)$ and $(1-y, 1-x)$ are symmetric w.r.t. the diagonal connecting points $(1,0)$ and $(0,1)$ (see Figure 3 ). As a result, curve $u_{\gamma}^{\mathrm{MMV}}$ is symmetric w.r.t. this diagonal. Note that $u_{\gamma}^{\mathrm{AD}}$ (Section 3.2) satisfies a similar property as it is also symmetric w.r.t. diagonal $(a, d)-(b, c)$.

Moreover, curve $u_{\gamma}^{\mathrm{MMV}}$ intersects the diagonal $(1,0)-(0,1)$ at a point with coordinates $(\beta, 1-\beta)$, with $1-\beta=u_{\gamma}^{\mathrm{MMV}}(\beta)$. Hence $\left(1-\beta^{\frac{1}{\gamma}}\right)^{\gamma}=\beta$, i.e. $\beta=\left(\frac{1}{2}\right)^{\gamma}$.

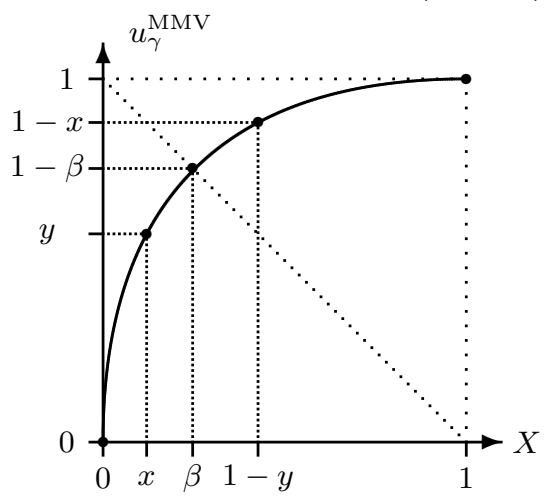

Fig. 3. Parametric function $u_{\gamma}^{\mathrm{MMV}}$.

The next result shows that Dichotomy method CEE can be used on interval $[0,1]$.

Lemma 4. Condition $\operatorname{Comp}(0,1)$ is fulfilled with $u_{\gamma}^{\mathrm{MMV}}$.

Given any $\bar{x} \in(0,1)$, one can easily find by dichotomy or a Gradient method the value $\bar{\gamma}$ such that $\int_{0}^{1} u_{\bar{\gamma}}^{\operatorname{MMV}}(x) d x=u_{\bar{\gamma}}^{\operatorname{MMV}}(\bar{x})$. Moreover, the next lemma provides bounds on $\gamma$ given the comparison of the decision maker.

Lemma 5. For any $\bar{x} \in(0,1)$, there exists a unique $\bar{\gamma}>0$ such that $\int_{0}^{1} u_{\bar{\gamma}}^{\mathrm{MMV}}(x) d x=$ $u_{\bar{\gamma}}^{\operatorname{MMV}}(\bar{x})$. Moreover, $\langle 1,[0,1]\rangle$ is less preferred (resp. preferred or indifferent) to $\bar{x} \in$ $[0,1]$, iff $\gamma>\bar{\gamma}$ (resp. $\gamma<\bar{\gamma}$ or $\gamma=\bar{\gamma})$.

\section{Related works}

The elicitation process based on standard gambles has been enriched in different ways. It has been used in AI as a baseline technique to elicit elaborate models such as the Generalized Additive Independence (GAI) model [9,3]. If the set of possible utilities is $U$, the decision rule can be the expected expected utility where the expectation is taken over the set of outcomes but also over the space $U$ of possible utility functions [2]. The probability over utilities is updated during elicitation using Bayes' rule in [4]. Standard gambles are used to elicit an imprecise utility in the framework of multi-attribute utility theory in [7].

It has been noticed in the litterature that the elicitation of the utility using standard gambles may result in wrong assessments of $u$ or in inconsistencies. Experiments indeed indicate that human beings as subject to a number of biases that distort their 
judgment about the perception of uncertainty. A canonical list of biases can be found in [12]. For further references, see also $[14,11]$. The most commonly encountered biases are: (1) probability weighting (individuals do not treat probabilities linearly, and tend to overestimate small objective probabilities, and under-estimate large ones [20]); (2) loss aversion (individuals are more sensible to losses than to gains) $[13,17]$.

Under the Expected Utility model, risk averse individuals (they prefer for instance a sure outcome $x$ to the gamble $\left\langle\frac{1}{2}, 0 ; \frac{1}{2}, 2 x\right\rangle$ ) are represented by concave utility functions. However, it has been noticed that the standard gamble method tends to exaggerate the concavity of the utility function to capture risk aversion [10]. Rank dependent expected utility treats the probability weighting bias by transforming the probability with a distortion function [15]. This model is generalized in the prospect theory, where gains and losses (demarcating a neutral level) are handled differently [13, 17]. Prospect theory models the two biases.

\section{Conclusion}

We have proposed in this paper the elicitation of a utility function over a set $X$ by comparing a gamble proposing $x$ in an interval $[a, b] \subseteq X$ with equiprobability, to a sure outcome $\bar{x} \in X$. This is a generalization of the elicitation process based on standard gambles. A consistency condition called Comp has been defined: it tells that for any $\bar{x} \in[a, b]$, there shall exist a value $\gamma$ of the parameters such that $\langle 1,[a, b]\rangle$ is indifferent to sure outcome $\bar{x}$. The piecewise affine model $u_{\gamma}^{\mathrm{PA}}$ and power model $u_{\lambda}^{\text {pow }}$ do not fulfill this condition. We propose the use of two models that fulfill this condition. The first one $u_{\gamma}^{\mathrm{AD}}$ is piecewise affine with an intermediate point where both the abscissa and ordinate numbers varies at the same time on a diagonal. This pattern can be repeated several times in adjacent intervals (for instance, in $\left[x_{1}, x_{2}\right],\left[x_{2}, x_{3}\right]$, etc). The second one is the MinMaxVar function $u_{\gamma}^{\mathrm{MMV}}$, which shares a symmetry property with $u_{\gamma}^{\mathrm{AD}}$. In both cases, we can derive constraints on the parameters of the utility model from any comparison of gamble $\langle 1,[a, b]\rangle$ to the sure outcome $\bar{x}$. Risk aversion occurs when $\langle 1,[a, b]\rangle$ is strictly less preferred to sure outcome $\frac{a+b}{2}$. Under our models, this implies concavity of utility functions. Similar results are obtained with standard gambles.

We can extend this work in several directions. We can extend the expected utility model that we used to represent some cognitive bias. One can think of rank dependent expected utility which is based on the Choquet integral. One can also think of other types of uncertainties, such as the reliability of sources. One would then compare making a decision from a source with low reliability providing a value $x \in X$ to another source with high reliability providing another value $x^{\prime} \in X$. We can also think of other models for information $[a, b]$, such as the set of probabilities having this support.

\section{Acknowledgments}

The first author was supported by the European project FP7-SEC-2013-607697, PREDICT "PREparing the Domino effect In crisis siTuations".

The authors would like to thank an anonymous review for helpful comments. 


\section{References}

1. C. A. Bana e Costa, J. De Corte, and J.-C. Vansnick. MACBETH. International Journal of Information Technology and Decision Making, 11:359-387, 2012.

2. C. Boutilier. On the foundations of expected expected utility. In Proceedings of the Eighteenth International Joint Conference on Artificial Intelligence (IJCAI-03), pages 285-290, Acapulco, 2003.

3. D. Braziunas and C. Boutilier. Minimax regret based elicitation of generalized additive utilities. In Proceedings of the Twenty-third Conference on Uncertainty in Artificial Intelligence (UAI-07), pages 25-32, Vancouver, 2007.

4. U. Chajewska, D. Koller, and R. Parr. Making rational decisions using adaptive utility elicitation. In Proceedings of the Seventeenth National Conference on Artificial Intelligence (AAAI-00), pages 363-369, Austin, TX, 2000.

5. A. Cherny and D. Madan. New measures for performance evaluation. Review of Financial Studies, 22:2571-2606, 2009.

6. B. de Finetti. Theory of Probability: A Critical Introductory Treatment. Wiley, 1974.

7. M. Farrow. Sample size determination with imprecise risk aversion. In 8th International Symposium on Imprecise Probability: Theories and Applications (ISIPTA'2013), Compiègne, France, July 2013.

8. M. Farrow and M. Goldstein. Almost-pareto decision sets in imprecise utility hierarchies. Journal of Statistical Theory and Practice, 3:137-155, 2009.

9. C. Gonzales and P. Perny. GAI networks for utility elicitation. In Proceedings of the 9th International Conference on the Principles of Knowledge Representation and Reasoning (KR), pages 224-234, 2004.

10. J. C. Hershey and P. J. H. Schoemake. Probability versus certainty equivalence methods in utility measurement: Are they equivalent? Management Science, 31:1213-1231, 1985.

11. R. Hogarth. Cognitive processes and the assessment of subjective probability distributions. Journal of the American Statistical Association, 70:271-294, 1989.

12. D. Kahneman, P. Slovic, and A. Tversky. Judgment under uncertainty: Heuristics and biases. Cambridge University Press, 2001.

13. D. Kahneman and A. Tversky. Prospect theory: an analysis of decision under risk. Econometrica, 47:263-291, 1979.

14. M. Morgan and M. Henrion. Uncertainty: A Guide to Dealing with Uncertainty in Quantitative Risk and Policy Analysis. Cambridge University Press, New York, 1990.

15. J. Quiggin. Generalized Expected Utility Theory: the rank-dependent model. Kluwer Academic, 1993.

16. T. Seidenfeld, M. Schervish, and J. Kadane. A representation of partially ordered preferences. Ann. Stat., 23:2168-2174, 1995.

17. A. Tversky and D. Kahneman. Advances in prospect theory: cumulative representation of uncertainty. J. of Risk and Uncertainty, 5:297-323, 1992.

18. J. von Neumann and O. Morgenstern. Game Theory and Economic Behavior. Princeton University Press, Princeton, NJ, 1953.

19. D. von Winterfeldt and W. Edwards. Decison Analysis and Behavioral Research. Cambridge University Press, Cambridge, 1986.

20. P. Wakker and A. Stiggelbout. Explaining distortions in utility elicitation through the rankdependent model for risky choices. Medecine Decision Making, 15(2):180-186, 1995.

21. P. Walley. Statistical Reasoning with Imprecise Probabilities. Chapman and Hall, London, 1991.

22. P. Walley. Towards a unified theory of imprecise probability. International Journal of Approximate Reasoning, 24:125-148, 2000. 